\title{
FUNDACIÓN Y TRADICIÓN DE LA MODERNIDAD LITERARIA EN VENEZUELA
}

POR

Víctor Bravo

PERFIL DE LA MODERNIDAD

La modernidad envuelve a la cultura latinoamericana y le otorga la conciencia del distanciamiento. Desde el interior de lenguas europeas transplantadas, según expresión de Octavio Paz, el continente nombra una modernidad que le es a la vez, y quizás de manera irremediable, interior y extraña. La experiencia de esa extrañeza, vivida sin embargo como lo que nos constituye en el fragor de una permanente heterogeneidad, nos lleva a la asunción y al rechazo: nuestra fundación nos niega y nuestra negación nos funda. Ángel Rama ha expresado esta contradicción de la cultura latinoamericana: "La modernidad no es renunciable y negarse a ella es suicida; lo es también renunciar a sí mismo para aceptarla".

¿Cuál es la experiencia latinoamericana de la modernidad? En los planos económico, político y social ha sido sin duda una historia de búsquedas, extravíos y fracasos: las estrategias de progreso, industrialización y democratización revelan, al final de siglo, sus imperfecciones o su ceguera. La reflexión sociológica ha dado cuenta de la distancia, al parecer cada vez más amplia, y a pesar de esfuerzos y logros significativos en uno y otro país, entre nuestras naciones y las naciones con un proceso intenso de modernización.

La modernidad cultural y literaria quizá podría resumirse en la palabra reflexividad. Según Edgar Morín, el paradigma que fundamenta la época moderna es el método cartesiano: la duda y negación de lo presupuesto o instituido y la búsqueda de una realidad o de una verdad esencial; doble movimiento: la negatividad que, como señalara Habermas, la lleva a rebelarse contra todo lo que es normativo, y la reconstrucción, el brillo de una promesa de felicidad, la revelación de que la vida, como indicara Musil, es de naturaleza utópica. En este sentido la conciencia reflexiva que la modernidad conlleva, interroga y cuestiona incesantemente las presuposiciones del existir: el ser y la verdad, el tiempo y la causalidad, el espacio y el sentido.

El ser, desprendido de la certeza religiosa, en el proceso de secularización que se inicia con el Renacimiento, encuentra, primero, con Descartes y la Ilustración, su centro en la racionalidad para, cuando ésta se pone en cuestión, enfrentarse a sus propios abismos del inconsciente o de la locura, de las infinitas formas de la alteridad que, sin embargo, alcanzan en el lenguaje su centro. Freud, y Nietzsche, Mallarmé y Heidegger fundarán, desde diferentes perspectivas, ese ámbito de lenguaje como "casa del ser", donde se 
diluyen y se reconstruyen todas las formas. Desde este ámbito, la verdad empieza a ser cuestionada en sus procesos de legitimación y ataduras con la jerarquía y el poder; el tiempo muestra una complejidad mayor que la flecha de su sucesión y el determinismo de la causalidad da paso a otras lógicas que sumen el sentido común en la perplejidad.

La modernidad nos enfrenta a la dimensión negativa de lo real, y el arte y la literatura se convierten en sus más claros expedientes: la belleza, esa gran certeza del arte que dominó la estética por veintidós siglos, se ve contaminada, a partir sobre todo del romanticismo, por las formas espesas y ásperas de la fealdad; la unidad de la obra, por la mutiplicidad y la heterogeneidad como poderosas imantaciones del sin sentido; y aparece, como expresión de la reflexividad, la percepción irónica del mundo como dualidad, como incongruencia. En la escena narrativa esa percepción irónica se presenta en procesos textuales que parten de la identidad o de la diferencia entre lenguaje y mundo: en el primer caso, la parodia es a la vez degradación y homenaje de textos que devienen intertextos, en una dialéctica de la degradación y recuperación del objeto parodiado, y lo grotesco se presenta como la aparición, festiva o monstruosa, de lo corporal; en el segundo, la paradoja como la posibilidad de engendramiento de una literatura que refuta el mundo en el acto mismo de construir mundos imposibles, y lo absurdo como la asunción del sin sentido y de lo insólito como otro signo de lo real que revela de este modo sus secretas zonas de la alteridad, de lo dual. Procesos irónicos de la incongruencia que derivan muchas veces hacia el humor y/o la alegoría. Con Cervantes y Rabelais los procesos irónicos de la modernidad entran en escena narrativa; $y$, como señalara Habermas, con el romanticismo se formularán como propuesta estética, asumirán claros contornos en la obra de Baudelaire, y se desplegarán luego en varios movimientos de vanguardia.

\section{AmÉrica LATINA Y LA MODERNIDAD LITERARIA}

¿Cómo se inscribe América Latina en ese amplio espectro de la modernidad literaria? Sin duda que no a través de lo que Octavio Paz llamara "la pobreza de nuestro romanticismo". Los procesos de negatividad y reconstrucción que el romanticismo realiza en Europa, Paz los observa en el modernismo dariano: "El modernismo - afirma - fue nuestro verdadero romanticismo". Si el romanticismo nuestro fue pobre inscripción y reflejo de las revolucionarias propuestas por los romanticismos europeos, el modernismo se presenta como un movimiento renovador de la cultura y el lenguaje; y podríamos decir que su cosmopolitismo, su esteticismo, su conciencia de las posibilidades expresivas del lenguaje, fundan una resonancia y abren las primeras puertas de la modernidad en el continente. Sin embargo, es posible decir que el movimiento abrió y cerró, como una luz que alcanza su mayor intensidad y se apaga, una posibilidad del amplio espectro reflexivo de lo moderno, que se va a expresar a plenitud, a nuestro juicio, fundamentalmente a partir de la década del veinte, como expresión silenciosa y compartida en diversos países, a veces coincidiendo con la vanguardia, pero manifestándose en extraños y solitarios escritores que escribirán en contextos donde las dominantes de recepción serán otras, y cuyas propuestas estéticas, en correspondencia con las más complejas expresiones de la modernidad, no gravitarán realmente en nuestra cultura sino después de la década del sesenta, cuando una nueva sensibilidad de recepción comienza a manifestarse. Estos 
escritores, llamados por Ángel Rama los olvidados, se van a constituir en los fundadores de una tradición de la modernidad en el continente, nuestra "tradición de la ruptura", según la paradojal expresión de Paz.

\section{FUNDACIÓN Y TRADICIÓN DE LA MODERNIDAD EN LA LITERATURA VENEZOLANA}

Quizás sea posible, desde la perspectiva de la literatura venezolana, observar algunos rasgos de la modernidad que hemos reseñado. Procesos textuales de la modernidad pueden ser observados como intencionalidad estética de algunos escritores venezolanos, cuyas obras pueden ubicarse de manera global a partir de la década del veinte. En este sentido nos permitimos deslindar por lo menos cinco núcleos fundamentales que, a nuestro juicio, abren el tratamiento irónico de los inicios de la modernidad en la literatura venezolana: 1) Lo disonante y lo grotesco; 2) La presencia de una "poética" del mal; 3) Lo absurdo y lo fantástico; 4) Los juegos de la temporalidad y la indeterminación; y 5) El problema de la verdad y el sujeto.

\section{Lo disonante y lo grotesco}

En 1922 José Rafael Pocaterra da a conocer sus Cuentos grotescos, y en 1924 Antonio Arráiz publica Áspero. En estos textos lo disonante y la deformación rompen con el tono celebratorio de nuestra literatura y se proponen articular un sentido estético desde el centro mismo de la negatividad. En Cuentos grotescos la deformación y la crueldad se contraponen en equilibrio ético y estético con las salidas morales que los cuentos proponen. Podría considerarse a José Rafael Pocaterra como el iniciador del cuento moderno en Venezuela. Su feroz e hiperbólico realismo atravesado por las vertientes contradictorias de la ironía y la enseñanza moral, la exploración en la fealdad de la condición humana, y en ese sentimiento de la fealdad que es la crueldad, oponen su propuesta estética a los tonos e inflexiones dominantes en la época, sumergida en la sensibilidad modernista de Manuel Díaz Rodríguez y Blanco Fombona, y atraída por el criollismo positivista de las primeras obras de Gallegos. Textos los de Pocaterra que se proponen como disonancia y ruptura y que abren el relato hacia un campo de negatividades, en consonancia con la estética de lo feo que ya había planteado Karl Rosenkranz en 1953, y que se va constituir en una de las recurrencias centrales de la estética de la modernidad. Sus cuentos nos revelan una clara conciencia de su propia constitución, expresada fundamentalmente en el complejo tramado de la enunciación que divide los textos en fragmentos contrastantes donde las afirmaciones y asunciones de la verdad se ven permanentemente refutadas 0 tachadas, en una dialéctica que es también la de la crueldad y del más alto sentido ético, y que revelan, en la topología de lo alto y lo bajo donde lo sublime es permanentemente degradado, diferentes caminos del relato para la restitución de la condición humana. Señalaremos tres de esas posibilidades en el meandro narrativo de los Cuentos grotescos: 1. La puesta en escena de lo feo unido a la crueldad, que es refutada, en una segunda instancia, por el juego de enmascaramientos del sentido logrado en la estructura de la enunciación, que contrapone, inesperadamente, el sentimiento positivo del amor que hace brotar la belleza en el seno mismo de la más extrema fealdad. Cuentos como "Las 
Linares", "Oropéndolas" o "La casa de la bruja", por ejemplo, recorren esta posibilidad estética y ética del relato; 2. La degradación ética de falsos valores, que son develados como tales por la composición irónica de los textos. Cuentos como "Bastón puño de oro" o "La llave", confluyen en estas características; y, 3. La ironía ante la crueldad social, más lejana del dictamen moral y más cercana de la dimensión trágica de algunas vidas humanas. Es en esta vertiente donde, a mi juicio, Pocaterra logra sus mejores y más vigentes textos. Cuentos como "La I latina" o "Panchito Mandefuá" ejemplifican este hallazgo estético que une ironía y humor negro y que hace de la escritura del lenguaje la huella de la crueldad y el desamparo.

Los textos disonantes de José Rafael Pocaterra se explican también en el contexto socio político de enfrentamiento a la dictadura de Juan Vicente Gómez, sobre cuyo período el autor dejó uno de los testimonios más importantes no solamente de Venezuela sino de todo el continente: Memorias de un venezolano de la decadencia (1927).

Dos años después de la publicación de Cuentos grotescos, en 1924, Antonio Arráiz publica su primer poemario, Áspero, donde continuará, por otros caminos, el espectro estético de la disonancia y la fealdad abierto por Pocaterra. En este texto, el canto celebratorio de América, de la patria, de la mujer, de la virilidad, se encuentra permanentemente atravesado por la negatividad de un discurso intencionalmente antipoético y donde aquellos valores son negados por medio del envés inesperado de la ironía. Poemas como "El hermano muerto", que contrasta el valor de la virilidad con su negación; o "Ancestral", donde el mito poético de la amada revela la asunción monstruosa de la sumisión amorosa: poemas que nos revelan la permanente y contradictoria confluencia de la exaltación y la disonancia, que crean el ritmo especial de esta poesía. Arráiz nunca abandonará el tono «áspero» de sus poesía, pero en posteriores poemarios como Parsimonia (1932), Cinco sinfonias (1939) y Preludios (1945), tal disonancia, en correspondencia con las búsquedas estéticas de la modernidad, se articulará en una «incongruencia» que es una propuesta estética, con las estructuras armónicas de la música.

\section{Lo absurdo y lo fantástico en la reflexividad festiva de la parodia}

La tienda de muñecos, de Julio Garmendia, publicada en 1927, parece abrir otra vertiente, en nuestro país, del discurso moderno: lo fantástico y lo absurdo unidos al humor y a la reflexividad festiva de la obra literaria ante su propia producción. En el prólogo que Jesús Semprum escribe para la primera edición del libro, afirma que Garmendia no tiene antecedentes en la literatura venezolana. Hoy podemos afirmar, con Ángel Rama, que en el momento de la aparición de La tienda de muñecos, Garmendia se inscribe en una amplia familia latinoamericana que entiende la literatura como una elegante e insólita manera de profundizar la fisura entre la literatura y la vida; familia a la que pertenecen sin duda Julio Torri en México, Graça Aranda en Brasil, Macedonio Fernández en Argentina y Felisberto Hernández en Uruguay.

Quizás en "El cuento ficticio" tengamos nuestro primer texto de ficción que tiene como referente su propia reflexividad. La condición del héroe que, como un nuevo Quijote, realiza la travesía para rescatar una vez más el carácter verdadero de lo imaginario, y la vindicación a la vez apasionada y paródica de lo ilusorio en la ficción, hace de "El 
cuento ficticio" nuestro primer manifiesto de la modernidad. Quizás nunca antes como en "La tienda de muñecos", "La realidad circundante" y "El librero", la ficción venezolana había abierto las compuertas que unen y separan lo real y lo imaginario, uniendo a lo absurdo esa forma leve del humor que a falta de otra palabra llamaremos perplejidad. Quizás, como en "El alma" y "El difunto yo", la pantalla de la subjetividad fue testigo de la insólita presencia del otro, que aparece allí para escenificar el despojo y el engaño que hacemos de nosotros mismos. En "El alma" la presencia del diablo nos coloca una vez más en esa tradición del pacto demoníaco que adquiere rasgo de modernidad con Goethe, que se ha abierto en infinidad de variantes desde entonces, y que en el cuento de Garmendia, como en "El alguacil alguacilado" de Quevedo, hombre y diablo miden sus propios engaños demoníacos. En "El difunto yo", el absurdo del otro que es "yo" sin serlo, se convierte a la vez en un absurdo lingüístico que desborda hacia el humor. Con la publicación de La tienda de muñecos lo fantástico, el absurdo y la levedad del humor abren el horizonte de una magistral literatura que ha tenido pocos continuadores en el país.

\section{Una poética del mal}

La publicación, en 1925, de La torre de timón y, en 1929, de El cielo de esmalte y Las formas del fuego, de José Antonio Ramos Sucre, introduce en nuestra literatura, primero, el canto celebratorio del mal, tal como aspiraba Baudelaire que debía hacer la poesía llamada por él moderna, y, segundo, la consciencia de la escritura como lugar del exilio, como refugio del ser, estremecido ante las furias desgarradoras de la vida, y de la muerte. La poesía de Ramos Sucre plantea, una vez más, en el contexto de la literatura moderna, la paradoja de una escritura que crea, de manera apasionada, otro mundo, en la herida misma de su ansia por el mundo. La poesía toda de José Antonio Ramos Sucre es canto a la muerte, más no canto celebratorio sino trágico. Nunca antes como en esta poesía, en la literatura venezolana, se había cantado el amor a la vida en el instante mismo de su abandono:

Yo quisiera estar entre vacías tinieblas, porque el mundo lastima cruelmente mis sentidos y la vida me aflige, impertinente amada que me cuenta amarguras.

Entonces me habrán abandonado los recuerdos: ahora huyen y vuelven con el ritmo de infatigables olas y son lobos aullantes en la noche que cubre el desierto de nieve.

Nunca en nuestro país y, quizás, en el continente, se había poetizado sobre el exilio como lugar del ansia y de la soledad; nunca antes se había invocado a la muerte como a la única salida desgarrada y fatal de un ser extraviado que ama la vida. Nunca como en la poesía de Ramos Sucre, el yo poético había reunido, en el centro mismo de su desgarramiento, el amor y la muerte, la belleza y la amargura, la fantasmagoría de la ciudad y la naturaleza, la poética de la angustia y el dolor; nunca el yo se había desdoblado para destruir al otro en el acto de destruirse a sí mismo; nunca antes el discurso poético en nuestro país había sido mostrado en su más alta plenitud y su más extrema insuficiencia; nunca antes la poesía había sido tan necesaria y tan inútil: la escritura como aplazamiento 
de la destrucción final se hace también travesía hacia la muerte, que hace del suicidio del poeta su signo más consecuente y desgarrado. Quizás podríamos ver en César Vallejo, su contemporáneo, aunque con una distinta experiencia de lenguaje, una actitud similar ante la relación entre ser y mundo, y una misma intuición sobre el doloroso desprendimiento de la vida.

Lo grotesco y la irrisión, lo fantástico y lo absurdo, el mal y la imposibilidad de la escritura, dibujan con Pocaterra y Arráiz, Garmendia y Ramos Sucre, el primer mapa de los inicios de la modernidad en la literatura venezolana.

\section{Los juegos temporales y la indeterminación}

En Cubagua, la novela de Enrique Bernardo Núñez publicada en 1931, se nos revela un texto donde muchos de los hallazgos textuales de la narrativa latinoamericana de los últimos cincuenta o sesenta años se encuentra ya como rasgos de sus frases y de su arquitectura. La posibilidad de la expresión y de la presencia cultural de lo indígena en el contexto de la cultura occidental, tal cómo lo planteara años después José María Arguedas en Todas las sangres (1964); el proceso de transculturación que observamos en Paradiso (1966), de José Lezama Lima; la "poética de la frase", posible de observar en Los peces (1968) de Sergio Fernández, o Maytreya (1978), de Severo Sarduy; la confluencia del tiempo como columna de la composición del texto, como se ve en Semejante a la noche (1958), de Alejo Carpentier, se manifiestan con su resonancia poética, mutatis mutandis, en esa brevísima novela de 1931 que es una de las primeras expresiones, en el continente, de una narrativa que en las últimas décadas ha llamado la atención de editores, críticos y lectores de diversas partes del mundo.

La confluencia de «tiempos» que refluyen a la superficie del relato para entrelazar historia y leyenda, para reiterar, de manera excluyente o superpuestos, los sentidos de riqueza y esterilidad, de esplendor y decadencia, y para expresar "el alma de la raza" en su proceso de transculturación; la presentación de personajes que son a la vez alegorías y signos ambiguos de un destino, hacen de Cubagua una de nuestras obras de mayor modernidad.

Podría decirse que la concepción del tiempo que rige la composición de la novela se acerca a la que años después formularía explícitamente Carpentier como premisa de algunas de sus obras: "el hombre es el mismo en diferentes épocas". Es posible decir que éste es el principio de composición de Cubagua, que ata y articula todos sus sentidos. En la novela de Núñez esta identidad no sólo se da en el plano nominativo y del ser (nombres y personajes son los mismos en diferentes épocas históricas) sino también establece sus analogías con la naturaleza y el cosmos ("las rosas son iguales, diríanse las mismas adorantes rosas de hace millares de años, y las estrellas siguen brillando largo tiempo, aún cuando rueden yertas y mudas en el espacio"). Si todo desaparece (en lo estéril, en el olvido) y todo regresa, la primera metáfora es entonces la del anillo: este atará verdad y delirio, historia y mito, en la superficie breve y llena de resonancias del relato.

Sobre el transfondo histórico de la fundación, esplendor y abandono de Nueva Cádiz, una de las primeras ciudades en el Nuevo Mundo, levantada en la aridez misma de la 
falta de agua pero en el fragor de la riqueza de las perlas, la novela, trasponiendo simbólicamente los valores semánticos de riquezas y aridez, identifica el destino, en 1925, de Leiziaga (quien, sobre las ruinas que "sepulta" la historia quiere restituir el «esplendor» con una nueva riqueza: el petróleo) con el que Lampugnano en 1528. Este procedimiento textual se amplía a otros personajes: Nila Cálice, contemporánea de Leiziaga, es también la hija de Rimarina, un cacique tamanaco que muere asesinado; y Fray Dionisio recorre los dos estratos históricos (tiempo de la fundación de Nueva Cádiz, siglo XVI; presente del relato, siglo XX) como expresión de los vasos comunicantes que el relato pone en escena para "acarrear» los materiales de la historia en la concreción de un nuevo horizonte narrativo.

Establecido el procedimiento textual (que revela la perfecta composición de la novela) es abrumadora la confluencia de lo divergente, de lo disyuntivo: Fray Dionisio, "cojo", tendrá un solo pie en la "vida", pues andará con su propia calavera bajo el brazo (y será él mismo la máxima concreción de lo diverso creador: sacerdote cercano a las prácticas de hechicería, asimilará el esplendor de la raza indígena, será el guardián de Nila Cálice y llevará de la mano a Leiziaga para el encuentro con Vocchi, en el momento irradiante de la novela. Será a la vez "Fray y Dionisio"); personajes como Gonzalo de Ocampo o Antonio Cedeño vivirán en dos tiempos; el "alma de la raza" buscará su expresión a pesar de la esclavitud o en el extravío de los nuevos tiempos; la historia, la leyenda y el mito alcanzarán el espesor de un mismo símbolo en el viaje que, guiado por Fray Dionisio, hará Leiziaga a las catacumbas de Cubagua: encuentro, en un contexto onírico, de los tesoros de El Dorado, pero resguardados por la presencia y el rito de Vocchi (Dios hermano de Amalivaca, en la mitología tamanaca el protector del nuevo hombre que nace de los frutos, después del diluvio) y de Nila Cálice (la descendiente de la raza tamanaca); encuentro con Leiziaga, el descendiente de los conquistadores. Ese encuentro (que es, simbólicamente extravío y fusión de nuestra cultura) es un viaje a los infiernos, de donde es posible ascender en busca de una nueva síntesis, la del hombre americano. Quizás aquí se encuentre el "secreto de la tierra" que propone la novela: es en amalgama y en la confluencia de nuestras heterogeneidades donde podemos alcanzar nuestra gravitación cultural. Años después de la publicación de esta novela, un antropólogo cubano, Fernando Ortiz, llamaría "transculturación" a ese proceso que parece constitutivo a nuestra cultura.

Cubagua, en su brevedad, es, a la vez, novela ambigua y alegórica, histórica y metafísica, realista y poética; y todo en la mágica concisión de un estilo que la hace una de nuestras obras más vigentes en el contexto de la narrativa moderna latinoamericana.

\section{El problema de la verdad y el sujeto}

El problema del sujeto y de la verdad será planteado en 1926, en Ifigenia, de Teresa de la Parra. Michel Foucault ha observado que en el centro de la literatura moderna nace la pregunta ¿quién habla? En la carta o el diario de María Eugenia Alonso, el personaje de la novela, la pregunta se amplía en espectro de complejidad: ¿por qué se escribe? En el mismo título la respuesta atiende a la levedad, tal como podría ser entendida por Calvino: se escribe por el fastidio; si atendemos a la confesión del personaje tendremos otra respuesta: se escribe para decir la verdad. Y en esa verdad el yo desea ser "otro" 
que no es, y no desea ser ese otro que es. Un laberinto del deseo configura la textura de los signos en la novela donde los espejos, proliferantes, se constituyen también en una metáfora de la mediación literaria que, en una especie de bovarismo, orienta el deseo del personaje que ve en los otros el otro de su deseo, y en la alabanza, en la mirada y, finalmente, en la escritura misma, el "espejo" de la verdad. Pero la escritura es, como se dice magistralmente en la novela, una "huida en espera de hallarse", un espejo deformador: el personaje escribirá no para decir la verdad, su intención explícita, sino para ocultarla. La salida del laberinto era una falsa salida: María Eugenia, el personaje que escribe, realmente desea ser el otro que dice que no desea ser (si leemos con detenimiento, desde el comienzo de la novela, el rechazo de María Eugenia a un destino como el de la tía Clara, en el fondo esconde el deseo de asumir ese destino, pero en su dimensión feliz del matrimonio), y, para completar el juego de máscaras del deseo, no desea ser el otro que dice que desea ser (a pesar de sus actitudes, María Eugenia no desea ser, por ejemplo, como Mercedes Galindo; de allí que, ante la llamada del amante, al final de la novela, inventará los obstáculos que le imposibiliten la huida, la asunción del amor pasión); en esta doble faz aparece uno de los por qué de la escritura: el infinito juego de la verdad y la mentira, el diferimiento de la asunción del verdadero deseo, donde el ser, permanentemente, se disuelve y se constituye, y donde el deseo, a la vez desnudo y enmascarado, y en continua metamorfosis, se convierte en la génesis misma de la escritura.

Una literatura se abre con la pregunta de Nadja, que inicia la novela de André Breton, en 1928: ¿Quién soy?; cuatro años antes, en la novela de Teresa de la Parra, María Eugenia Alonso, el personaje, se había hecho esa pregunta, sin hacerla realmente de manera explícita, buscando el eco de la respuesta en la multiplicidad de espejos de la media luna de su armario, en el azogue de la escritura, en el elogio y la prohibición, en el mirar el éxtasis de la mirada que la mira, en las máscaras de la confesión.

La novela enfrenta, en la disyunción de lo masculino y lo femenino, el drama entre el poder y el deseo. El poder revela lo que podríamos llamar "el orden masculino", y se expresa, primero, en el espectro de prohibiciones que pesan sobre lo femenino: segundo, en lo económico, caracterizado por el despojo y la dependencia; $y$, tercero, en un sistema de inclusiones y exclusiones que permite la permanencia de ese orden: Eduardo y César Leal serán los guardianes de ese orden; la abuela y Clara lo integrarán, por sumisión. En este ámbito, el matrimonio es acto de coerción y dominio, y la acumulación será el rasgo distintivo de lo masculino; en otro ámbito, tres personajes, Pancho, Gabriel Olmedo y Mercedes Galindo, se opondrán al matrimonio para asumir el amor, y a la acumulación para asumir la disipación. Esa diferenciación de mundos será la que consiga María Eugenia, pero el orden masculino impondrá sus rigores y cubrirá en su proceso de integración a quienes no se le habían sometido: Mercedes Galindo asumirá finalmente la sumisión del matrimonio, viviendo su deseo de exclusión como tragedia; Pancho se resignará, viviendo su diferencia en la parodia y, finalmente, morirá; y la ambición perderá a Gabriel Olmedo, quien asumirá el engaño como conducta. El ansia de ruptura y libertad se vive así realmente como un sentimiento anteriormente derrotado por el orden masculino de la acumulación y las convenciones, y los personajes "descarriados" serán vencidos por el peso del orden. En María Eugenia esa doble dimensión del deseo será vivida de una manera compleja: deseando asumir la libertad, tal deseo, como su escritura, será una 
máscara, un diferimiento del verdadero y más oculto deseo: alcanzar lo que en tía Clara fue imposibilidad y despojo: el matrimonio. De allí que ante la llamada final del amante, María Eugenia invente la cadena de obstáculos que le impedirán el salto hacia la felicidad y/o el abismo, como ocurrió, en un momento estelar de las pasiones, a Ana Karenina, por ejemplo, y decide asumir el destino que verdaderamente la ata desde el principio del texto: la sumisión.

Ifigenia, de Teresa de la Parra, pone en evidencia la escritura como criba del deseo, como enmascaramiento de la verdad, y como juego de espejos donde a la vez aflora y se distancia el ser.

En 1962, Guillermo Meneses publicará El falso cuaderno de Narciso Espejo para replantearse, con diferentes tonos e inflexiones, el problema de ser y la verdad, y para revelar que en el camino de la escritura moderna, sólo es posible encontrar un juego de máscaras que nos llevan no al encuentro del ser o de la verdad sino, en el mejor sentido Nietzscheano, al encuentro con la nada.

La novela moderna se propone como un discurso negativo sobre los referentes del mundo: la parodia y el humor, la paradoja y el absurdo ponen en evidencia el envés de las certidumbres, los mundos representados en la asunción de las verdades y las certezas. La novela es una de las prácticas modernas de la dualidad del lenguaje, de su manifestación irónica. El primer referente ironizado por la novela moderna, lo sabemos, es la figura del héroe, como expresión narrativa de una de las negatividades centrales de la modernidad: el cuestionamiento del ser. "El héroe - dirá en este sentido Maurice Blanchot- es el don ambiguo que nos concede la literatura antes de tomar conciencia de sí misma". El cuestionamiento del ser presupone el cuestionamiento de la verdad, de allí que la novela moderna pueda ser vista como la escena del juego y del vértigo especular donde el ser y la verdad, para decirlo con una frase de Lacan, son por fin cuestionados.

El falso cuaderno de Narciso Espejo, transitando caminos narrativos apenas entrevistos por la literatura latinoamericana de su hora, lleva a límites inusitados esta propuesta estética. Construida por "expedientes", "legajos", "documentos", la novela se apropia, paródicamente, del discurso que tiene como función instaurar la verdad, el discurso jurídico, para iniciar el juego especular trenzado por el deseo, y donde el doble y la incesante negación del ser afirman la realidad de la imagen, que a la vez se niega en una cadena de tachaduras donde el sujeto enunciador finalmente se disuelve, se disemina, para que el texto hable desde el vacío. Juego de formas que abre una profunda indagación narrativa sobre la enunciación, la novela también ofrece ese vértigo de negaciones en el tramado del relato mismo, por medio de la causalidad de lo fantástico: la presencia de la nube amarilla, que produce, en correspondencia con aquel turbión de negatividades, la causalidad negativa del asesinato, el suicidio, y la negación de la "verdad" instaurada por la imagen. Juán Ruiz tachando su ser para que viva la imagen de Narciso Espejo, en "memorias" que, por falsas, niegan a su vez su condición de tales; Narciso Espejo afirmándose en esa especie de "novela de educación" que encontramos en el "Cuaderno C", la tachadura del Cuaderno $\mathrm{C}$ hecha por el personaje que bien puede llamarse Juán Pérez o tener otro nombre, la obsesión especular que rodea la figura de Lola Ortíz, etc., crean ese vértigo de reflejos que, no obstante, no se propone solamente como juego formal sino también como indagación de la endeble y secretamente vertiginosa condición 
de lo real. Así lo dirá Narciso Espejo, en una confesión sobre su imagen, que perfila la poética de la novela: "Suponer que esto es cosa de amores es absurdo. Suponer que yo me escondo para lograr el placer de mi carne y ver mi goce en los reflejos es igualmente tonto y perverso. Lo que yo busco en el agua es todas las preguntas a la que debo dar contestación". La literatura moderna, como este Narciso de la literatura venezolana, buscará en las aguas del lenguaje el juego de su composición y la revelación de las complejas y sorprendentes formas de lo real.

El falso cuaderno de Narciso Espejo abre en la literatura venezolana un modo del tratamiento narrativo que no será retomado sino a partir de la década del cincuenta, cuando los integrantes del grupo Sardio y, posteriormente, de Techo de la ballena, postulen la tradición de la modernidad en la literatura venezolana.

En 1873 Rimbaud afirmaba que "es preciso ser absolutamente modernos". Esta exigencia no empieza a tener clara respuesta en nuestro país sino hasta la década del veinte, en un grupo de escritores que hará de lo grotesco o de lo fantástico, del mal y de la indeterminación, del cuestionamiento del ser y de la verdad, su escudo y su crítica a lo real, y el tramado mismo de la escritura.

\section{LA GRIETA DE LA MODERNIDAD}

En Venezuela la modernidad no se "desplegará en la vanguardia". A diferencia de países como Chile, con Vicente Huidrobo, y México, con José Juan Tablada, donde la vanguardia se asume como hallazgo inusitado de la expresión, en Venezuela la vanguardia no se vive como grieta, como transgresión, como turbión de negatividades; se vive más bien como adscripción, como identificación tardía con un modo de asumir la expresión estética. Nuestra revista vanguardista más importante, válvula (1928), en su único número, reúne un grupo heterogéneo de escritores (Arturo Uslar Pietri, Carlos Eduardo Frías, Nelson Himiob, José Salazar Domínguez, Pedro Sotillo ...) que se adscribe a la vanguardia en un acto efímero que los nutre sólo como punto de partida para la búsqueda de sus propias posibilidades expresivas. Este modo de asumir la vanguardia es el mismo de los poetas agrupados en Élite (1928 a 1935) y Viernes (1937) sin realmente crear la grieta y la ruptura engendradora de nuevos lenguajes que en otras latitudes la vanguardia había creado. En Venezuela otros escritores (que a veces aparecen en las listas de los recuentos históricos que se hacen sobre uno u otro grupo), distintos distantes y contemporáneos, dibujaban sin embargo, de manera firme y secreta, el mapa inicial de una estética que décadas después se revelará como punto fundacional de nuestra modernidad.

El año de 1958 abre una grieta en Venezuela: grieta política y cultural que llena de estremecimientos al país. Junto a la instauración de un nuevo orden político que gesta a su vez el brote de la insurgencia, se origina también una incesante proclama de ruptura y fundación en el plano de la cultura. Se produce, como señala José Ramón Medina, "una evidente y vital interacción entre lo literario y lo político". El Grupo Sardio, que cumple una intensa actividad entre 1958 y 1961, cuando se disuelve para dar paso a un grupo más radical, Techo de la ballena, inicia esa decisión de ruptura con la tradición oficial de una literatura celebratoria ligada aún a las últimas manifestaciones del positivismo, y que tenía en Rómulo Gallegos su referencia emblemática. Se inicia la postulación de 
una práctica estética abierta a las resonancias de lo universal y a la conciencia crítica, convocando, en la filigrana misma de lo literario, esa función estética que Adorno veía en el texto moderno: la revelación de las formas de reificación entre los sistemas y la existencia vital. En el "Testimonio" que abre el primer número de la revista del grupo (mayo-junio 1958), se señala:

No confundimos universalidad con cosmopolitismo, pero se nos hace evidente que el exceso de color local, con todas sus derivantes, ha viciado de raíz gran parte de nuestras manifestaciones artísticas. Así como condenamos cualquier esteticismo, condenamos también cualquier nacionalismo exacerbado y arrogante .... Es imperioso elevar a perspectivas más universales los alucinantes temas de nuestra tierra.

La asunción de la cultura y el arte se convierte así en un acto de separación y negatividad, a la vez que un acto fundacional: "Nos sentimos asistidos por una nueva visión y una distinta sensibilidad", dirán los sardianos, indicando la separación de aguas que significaba su actitud. El grupo publicará ocho números de la revista Sardio donde la "nueva visión" y la "distinta sensibilidad" establecerán los parámetros para la asunción y la expresión de la cultura. El grupo igualmente editará algunos libros donde se perfilarán con claridad los rasgos de su propuesta estética: Estrechos son los navios, de Saint-John Perse, en traducción de Guillermo Sucre, que es uno de los signos indicativos de su ansia de universalidad; el libro de cuentos Las hogueras más altas (1957), de Adriano González León que, retomando las propuestas de Pocaterra y Arráiz, explora las posibilidades expresivas de lo grotesco; El reino (1958), de Ramón Palomares, que sitúa el verso en posibilidades inusitadas de expresión; Los pequeños seres (1959), de Salvador Garmendia, una de las novelas más importantes de las últimas décadas que, al decir de Juan Liscano, "rompe con el peso planetario de Rómulo Gallegos"; y Fantasmas y enfermedades (1961), de Francisco Pérez Perdomo que, con una profunda originalidad, realiza una suerte de reescritura del universo poético legado por José Antonio Ramos Sucre. En la década del sesenta diversos grupos y revistas serán la expresión de ese fragor de rupturas y fundaciones; Sardio, como indicáramos, da paso a Techo de la ballena, grupo más radical que romperá de manera violenta con los patrones formales de la belleza y propondrá una estética de lo feo, en la más rica tradición de lo corporal y de la fealdad, que viene del romanticismo. En este grupo confluyen poetas, narradores, y artístas plásticos que no sólo plantearán, como en Sardio, de donde proceden muchos de ellos, lo feo como una de las escandalosas concreciones de la manifestación estética; profundizando en esta actitud transgresiva, los artístas de El techo de la ballena avanzarán por caminos que luego serán propios de la posmodernidad: el escándalo de la disolución de las formas, de la negación misma de lo que Peter Bürger llama la institución arte. El arte no como búsqueda de la belleza sino, de manera radical, como búsqueda de lo informe. Sus exposiciones "Para restituir el magma" (1961), "Homenaje a la cursilería" (1961) y, sobre todo, la exposición de Carlos Contramaestre, "Homenaje a la necrofilia" (1962), llamada por Salvador Garmendia "poética del horror urbano", van a colocar la transgresión y la negatividad en la génesis misma de la expresión artística. En haa, grupo nacido en 1962 , que surgirá buscando un discurso universal que se oponga al localismo de la 
literatura del país; Tabla redonda, que someterá la búsqueda estética a la emergencia política .... Estos grupos y revistas reunirán a los escritores que en los siguientes años producirán la literatura más relevante del país: la poesía de Juán Sánchez Peláez y Ramón Palomares, de Guillermo Sucre y Rafael Cadenas; la narrativa de Adriano González León y Salvador Garmendia, de José Balza y Luis Britto García.

Es posible decir que Sardio y Techo de la ballena provocan la ruptura que profundizarán grupos posteriores: a partir de ese momento la modernidad como conciencia estética, como "pasión crítica", comienza a gravitar en tanto que signo fundamental de nuestra literatura, y sus propuestas textuales empiezan a ser asumidas como las expresiones más auténticas de lo estético. A partir de estos dos grupos se remueven las estructuras y presuposiciones de la producción y recepción de obras y la tradición heredada empieza a ser cuestionada, rechazada, en el mismo acto en que empiezan a destacarse, como antecedentes o génesis de una nueva tradición, aquellas obras que desde la década del veinte iniciaban los planteamientos reflexivos de la modernidad.

A partir de Sardio y Techo de la ballena la modernidad literaria venezolana inicia el proceso de recuperación de su tradición - proceso que no ha concluido-y traza los cauces para la literatura posterior; cauces de la reflexividad para la expresión de la literatura venezolana contemporánea. 